УДК 004.051

\title{
МОДЕЛИРОВАНИЕ И ОЦЕНКА ЭФФЕКТИВНОСТИ ИНТЕГРИРОВАННЫХ СИСТЕМ БЕЗОПАСНОСТИ ОБЪЕКТОВ, ПОДЛЕЖАЩИХ ОБЯЗАТЕЛЬНОЙ ГОСУДАРСТВЕННОЙ ОХРАНЕ
}

\author{
В. А. Дурденко ${ }^{\star}$ А. А. Рогожин ${ }^{\star *}$, Б. О. Баторов ${ }^{\star * *}$ \\ ${ }^{*}$ Воронежский государственный университет \\ ${ }^{*}$ Краснодарский университет МВД России \\ ${ }_{* * \star}$ Академия управления МВД России
}

Поступила в редакцию 21.05.2018 г.

\begin{abstract}
Аннотация. Предложены структурно-параметрические модели, позволяющие количественно оценить эффективность интегрированных систем безопасности объектов, подлежащих обязательной государственной охране. Для проведения вычислительного эксперимента использован общий логико-вероятностный метод и технология автоматизированного структурно-логического моделирования. В статье разработана технологическая дорожная карта процесса управления комплексной безопасностью объектов, подлежащих обязательной государственной охране.

Ключевые слова: структурно-параметрическая модель, оценка, эффективность, интегрированная система безопасности, объекты, подлежащие обязательной государственной охране.

Annotation. The structural-parametric models allowing to quantify efficiency of the integrated security systems of the objects which are subject to obligatory state protection are offered. For carrying out computational experiment the General logical and probabilistic method and technology of the automated structural and logical modeling is used.

Keywords: structural-parametric model, estimation, efficiency, the integrated security system, the objects, which are subject to obligatory state protection.
\end{abstract}

\section{ВВЕДЕНИЕ}

В настоящее время государственную охрану объектов, подлежащих обязательной государственной охране, уполномочены обеспечивать профессионально подготовленные и соответствующим образом оснащенные подразделения вневедомственной охраны войск национальной гвардии Российской Федерации, ФГУП «Охрана» Росгвардии, федеральной службы охраны и ведомственной охраны федеральных государственных органов.

Управление процессом обеспечения комплексной безопасности объектов, подлежащих 2018

() Дурденко В. А., Рогожин А. А., Баторов Б. О., обязательной государственной охране, неразрывно связано с использованием интегрированных систем безопасности (ИСБ) [1-3]. ИСБ представляют собой структурно-сложные технические системы и при их создании приходится использовать различные технические и программные средства, как по функциональному назначению, так и, возможно, агрегировать оборудование и программное обеспечение разных производителей.

На стадии проектирования (разработки) ИСБ закладываются все требуемые показатели качества, в том числе наиболее важные из них -показатели надежности и эффективности. Здесь необходимо учитывать, что оцениваемые структуры ИСБ могут быть одинаково высоконадежными, даже иметь 
Моделирование и оценка эффективности интегрированных систем безопасности объектов ...

одинаковые показатели надежности, но с разной эффективностью выполнять свою целевую функцию. При этом требуется решение научной задачи - оценки эффективности выполнения целевой функции конкретной структурой ИСБ.

Проведен анализ научных работ в предметной области. В [4-8] приведены результаты кратких обзоров существующих подходов, методик и критериев оценки эффективности информационных систем и технологий, а также систем физической защиты. Установлена неоднозначность используемых определений эффективности, и как следствие, сложности построения формализованной количественной методики ее оценивания. Учитывая вероятностную природу исходных данных, используемых для получения количественных оценок эффективности, сделан вывод о предпочтительности использования методов логико-вероятностного моделирования. Также в [9] автор приходит к выводу, что: «логико-вероятностные методы являются мощным механизмом анализа структурно-сложных систем. Их применение для оценки эффективности систем физической защиты таит в себе большие потенциальные возможности и должно найти себе достойное место в практике реального проектирования». Предложенный в [10] подход к оценке эффективности систем безопасности на основе метода Монте-Карло защищен патентами на территории Европы и Канады, однако более пригоден для оценки экономической эффективности проектируемой системы, поскольку позволяет вычислить стоимость системы, которая затем сравнивается с ранее заданной.

Очевидно, не существует универсального подхода к определению и оценке эффективности ИСБ как сложной технической системы. Так, при детерминистическом подходе эффективность понимается как степень выполнения требований по физической защите объекта и оценивается экспертным путем. При логико-вероятностном подходе эффективность формулируется как вероятность нахождения системы в безопасном состоянии в рамках построенного сценария развития опасности. При вероятностно-временном подходе под эффективностью понимается вероятность того, что резерв времени сил охраны окажется больше нуля.

В любом случае, для оценки эффективности ИСБ необходима разработка ее адекватной модели и проведение соответствующих вычислительных экспериментов.

Таким образом, для решения научной задачи оценки эффективности ИСБ объектов, подлежащих обязательной государственной охране, с позиции системного подхода необходимо разработать модель ИСБ, которая должна содержать в себе следующую информацию:

- о составе, структуре и межэлементных связях в ИСБ. При этом в роли элементов модели ИСБ выступают технические средства, оказывающие влияние на эффективность ИСБ;

- о количественных показателях надежности элементов ИСБ (как правило, вероятностных или временных показателях). Подразумевается, что если элемент работоспособен, готов к выполнению целевой функции, то он эффективно ее выполняет.

\section{РАЗРАБОТКА СТРУКТУРНО- ПАРАМЕТРИЧЕСКИХ МОДЕЛЕЙ ИСБ}

Традиционно поставленная научная задача может быть решена в два этапа:

- разработка структурной модели ИСБ, дающей общее представление о наиболее важных элементах и их взаимосвязях;

- преобразование имеющейся структурной модели в структурно- параметрическую.

Перейдем к решению данной задачи.

На первом этапе в качестве структурной модели будем использовать базовую структурную модель ИСБ, предложенную в [11]. Универсальность такой структуры ИСБ обусловлена наличием функционально необходимых элементов. Описание элементов базовой структурной модели ИСБ и выполняемых функций представлено также в [11]. Базовая структурная модель ИСБ позволяет перейти к разработке структурно-параметрической модели эффективности, позволяющей установить количественную связь между 
Условные обозначения взвешенного орграфа ИСБ

Таблица 1

\begin{tabular}{|c|c|c|}
\hline $\begin{array}{l}\text { № } \\
\Pi / \Pi \\
\end{array}$ & $\begin{array}{c}\text { Условное графическое } \\
\text { обозначение }\end{array}$ & Характеристика элемента \\
\hline 1 & & Вершина $i$ орграфа с весом $P_{i}-$ основная вершина \\
\hline 2 & & $\begin{array}{l}\text { Вершина } y \text { орграфа с весом } P(y) \text {, предназначенная для удоб- } \\
\text { ства построения, т. е. на выходе вершины, то же, что и на } \\
\text { входе - дополнительная вершина }\end{array}$ \\
\hline 3 & $\longrightarrow$ & Дуга орграфа с весом «ИЛИ» \\
\hline 4 & $\longrightarrow$ & Дуга орграфа с весом «И» \\
\hline 5 & 0 & Дуга орграфа с весом «НЕ-И» \\
\hline 6 & $\longrightarrow$ & Дуга орграфа с весом «НЕ-ИЛИ» \\
\hline
\end{tabular}

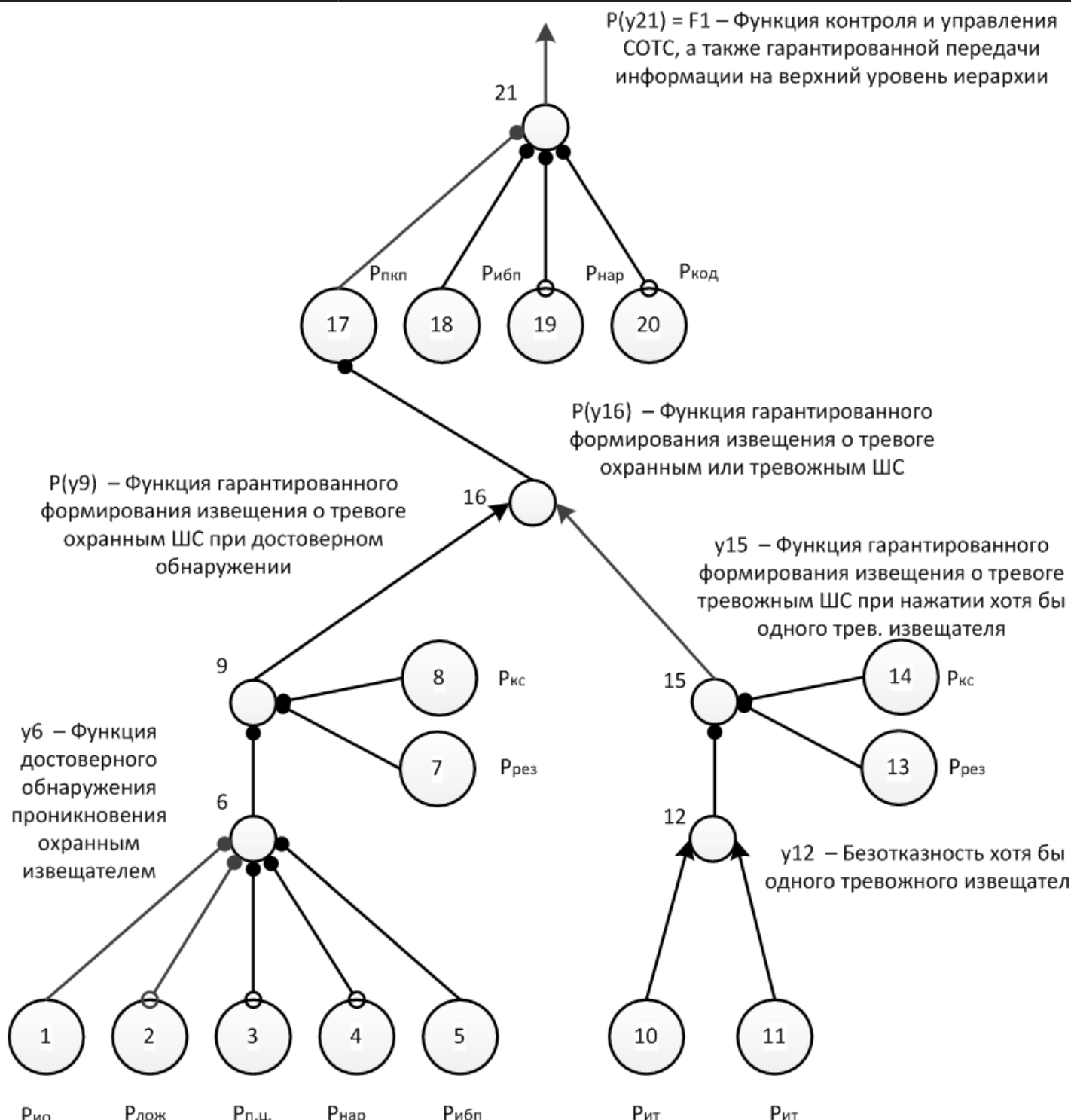

Рис. 1. Структурно-параметрическая модель подсистемы охранно-тревожной сигнализации СОТС ИСБ 
Моделирование и оценка эффективности интегрированных систем безопасности объектов ...

функциональными и вспомогательными параметрами системы.

Структурно-параметрическая модель ИСБ может быть представлена в виде взвешенного орграфа. Для его построения используем следующие условные обозначения элементов (см. табл. 1).

Структурно-параметрические модели подсистем ИСБ на уровне функционально необходимых элементов приведены на рис. 1-7. Структурно-параметрическая модель ИСБ приведена на рис. 8.

Формально взвешенный орграф, а следовательно, и структурно-параметрическая модель ИСБ представляются в виде следующей математической модели:

$$
G=(V G, E G, P V G, L E G),
$$

где $V G$ - множество вершин орграфа, причем вершинами являются бинарные события реализации/не реализации техническими средствами ИСБ своих функций, а также реализации/не реализации дестабилизирующих факторов, влияющих на надежность, эффективность ИСБ. При этом учет возможных дестабилизирующих факторов, в том числе воздействий нарушителя, подчеркивает большую адекватность модели и большую достоверность далее полученных результатов настоящего исследования. Таким образом, формально $V G=(I \cup Y)-$ множество вершин основных $I$ и дополнительных $Y$ (см. табл. 1);

$E G$ - множество дуг, определенных отношением $E$;

$P V G$ - веса вершин орграфа, которые определяются как вероятностные характери-

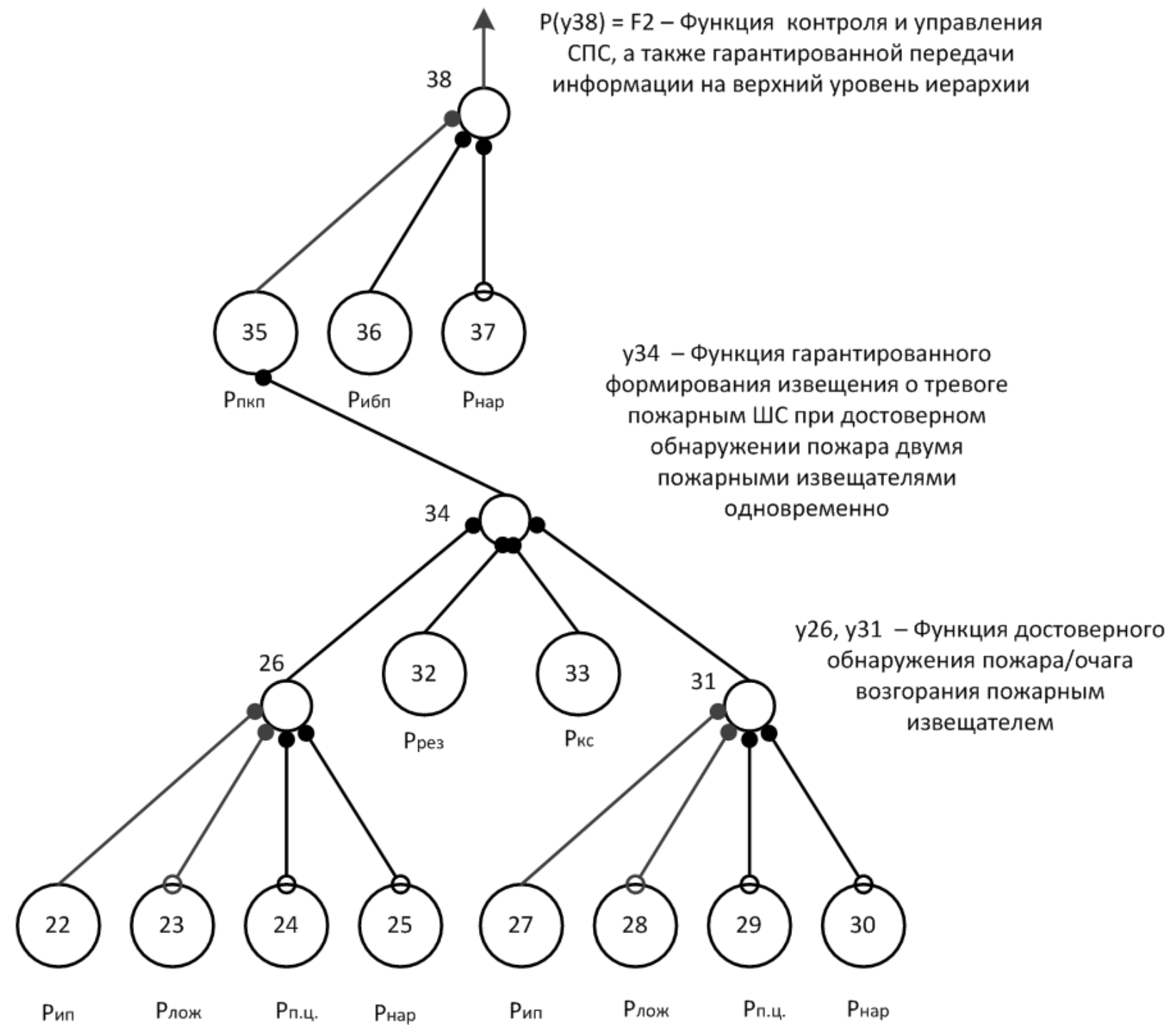

Рис. 2. Структурно-параметрическая модель подсистемы пожарной сигнализации СПС ИСБ 


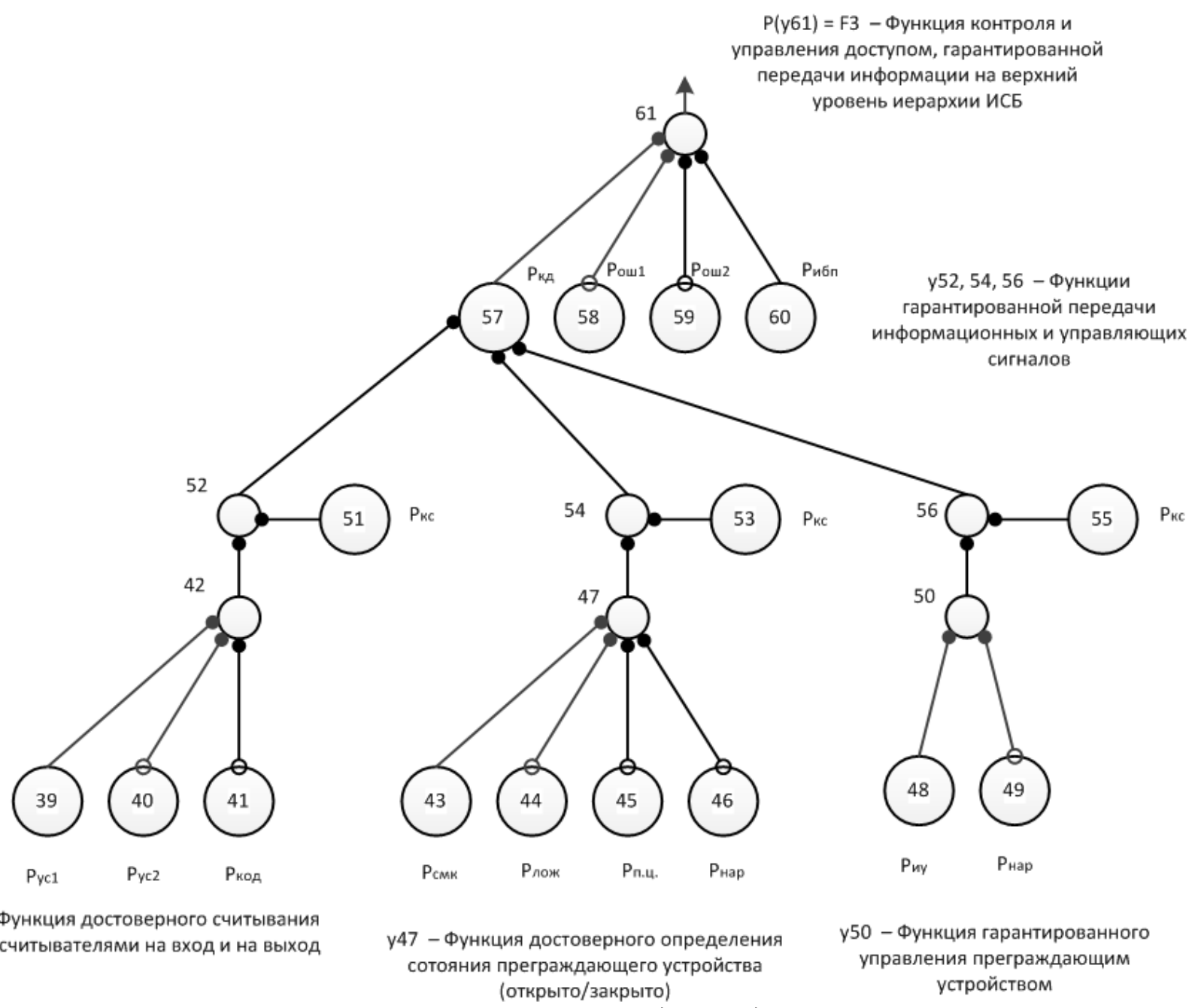

Рис. 3. Структурно-параметрическая модель подсистемы контроля и управления доступом СКУД ИСБ

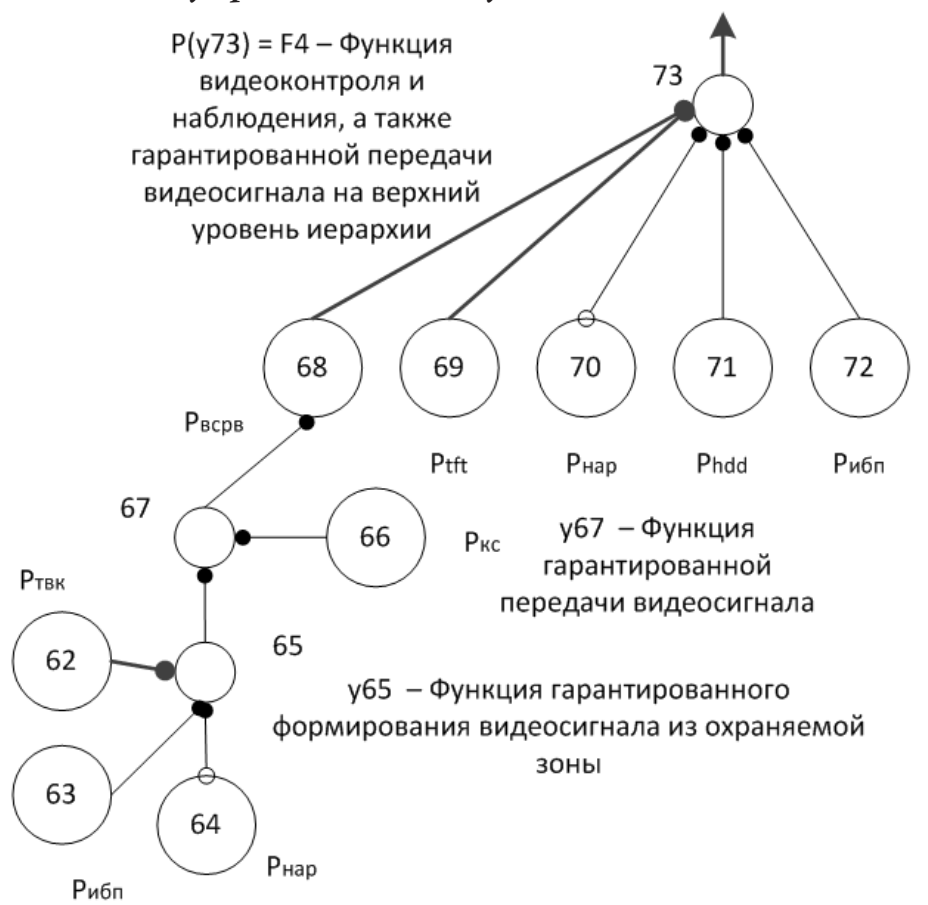

Рис. 4. Структурно-параметрическая модель подсистемы охранной телевизионной СОТ ИСБ 
Моделирование и оценка эффективности интегрированных систем безопасности объектов ...

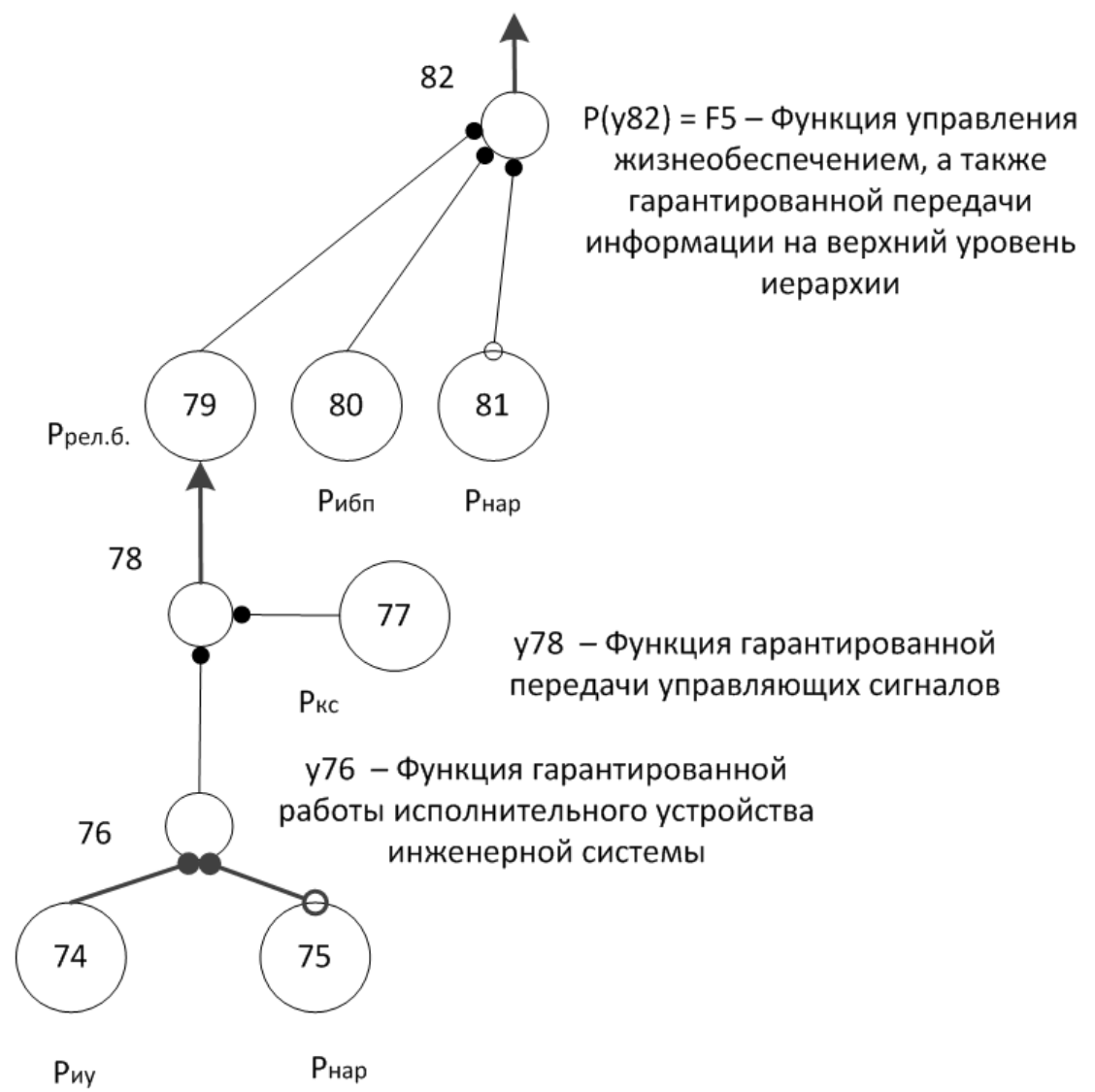

Рис. 5. Структурно-параметрическая модель подсистемы управления жизнеобеспечением СУЖ ИСБ

$$
\begin{gathered}
\mathrm{P}(\mathrm{y} 86)=\mathrm{F} 6-\text { Функция передачи } \\
\text { информации между } \\
\text { подсистемами в ИСБ }
\end{gathered}
$$

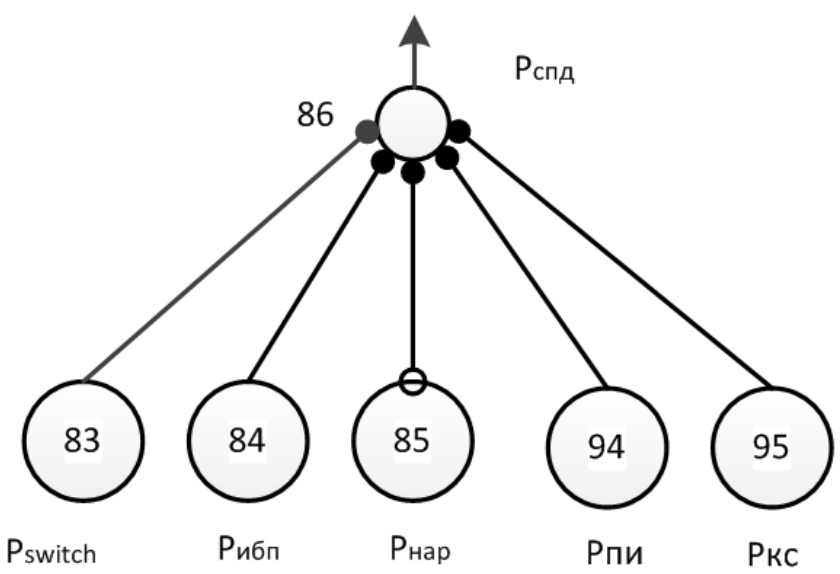

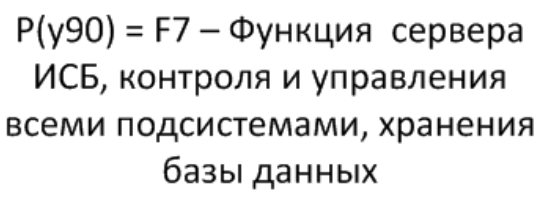

базы данных

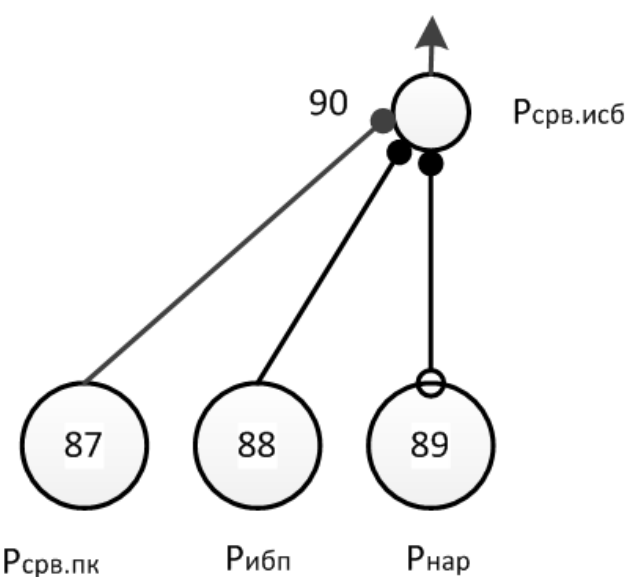

\section{Рибп Рнар}

Рис. 7. Структурно-параметрическая модель системы сбора и обработки инбормации (сервера) ССОИ ИСБ 


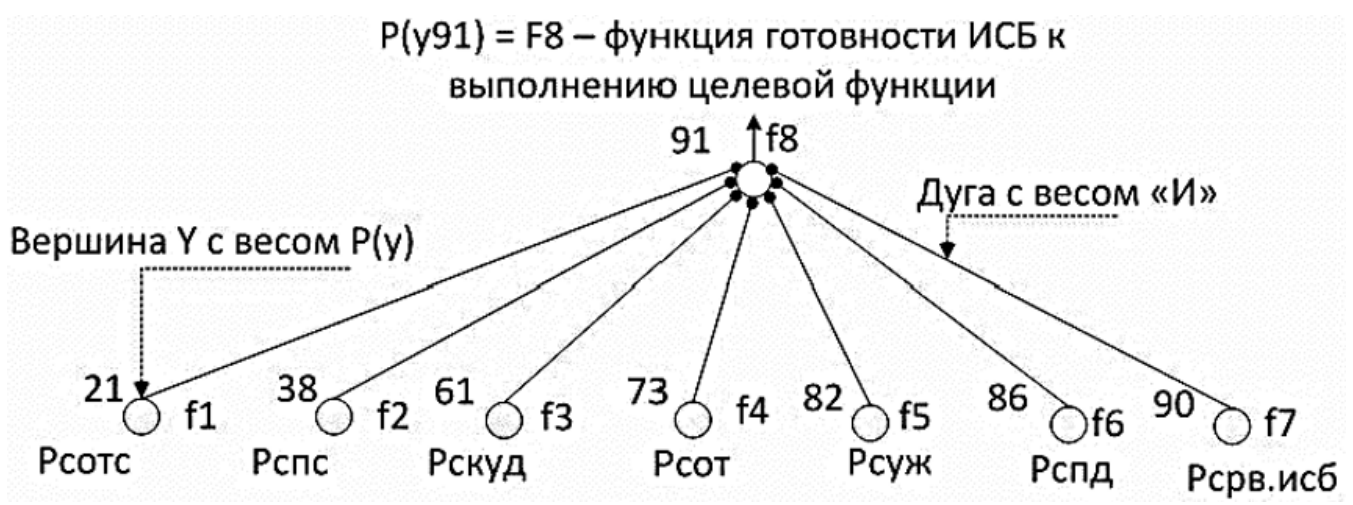

Рис. 8. Структурно-параметрическая модель ИСБ на уровне подсистем

стики, т. е. как вероятность $P_{i}$ реализации/не реализации элементом $i$ своей целевой функции (в качестве $P_{i}$, в зависимости от исследуемого режима функционирования ИСБ, а также от восстанавливаемости элементов, могут выступать коэффициент готовности элемента $i$ к выполнению целевой функции $K \Gamma_{j}$, вероятность безотказной работы элемента ИСБ - $\left.P_{i}(t)\right)$, т. е.

$$
P=\left\{P_{i}(t), K \Gamma_{j}\right\}, i \in N, \quad j \in W,
$$

где $N$ - множество номеров невосстанавливаемых элементов ИСБ,

$W$ - множество номеров восстанавливаемых элементов ИСБ,

$P_{i}(t)$ - вероятность безотказной работы невосстанавливаемых элементов ИСБ,

$К \Gamma_{j}-$ коэффициент готовности восстанавливаемых элементов ИСБ;

$L E G$ - веса дуг орграфа, которые задаются в зависимости от логических связей между элементами ИСБ с помощью логических операций И, ИЛИ, НЕ:

$$
L=\{И, И Л И, H E\} .
$$

Таким образом, была получена структурно-параметрическая модель ИСБ, которая очевидно является логико-вероятностной. То есть предложенная структурно-параметрическая модель ИСБ может быть преобразована в строгую логическую, а затем в вероятностную для последующего расчета показателей и оценки ее эффективности.

Воспользовавшись общим логико-вероятностным методом [12] и методикой построения немонотонных моделей эффективности систем [13], разработаем модель эффектив- ности ИСБ и произведем вычислительный эксперимент по определению показателя эффективности ИСБ с помощью программного комплекса «АРБИТР» [14].

Согласно [13]: «под реальной эффективностью $W$ качественно сложной системы понимается математическое ожидание значения показателя эффективности ее функционирования при случайном нахождении (по причинам неабсолютной устойчивости) в состояниях $Y_{j}$ полной и частичной работоспособности», «под структурной устойчивостью будем понимать комплексную характеристику способности системы сохранять (или не сохранять) работоспособность, определяемую различными совместными комбинациями свойств ее структурной надежности, стойкости и живучести».

\section{МОДЕЛИРОВАНИЕ И РАСЧЕТ КОМПЛЕКСНОГО ПОКАЗАТЕЛЯ УСТОЙЧИВОСТИ ИСБ}

На первом этапе построим модель (схему функциональной целостности - СФЦ) структурной устойчивости рассматриваемой ИСБ, декомпозированной до уровня подсистем (см. рис. 8).

Для этого:

1) заменим дополнительные вершины структурно-параметрической модели ИСБ на функциональные вершины аппарата СФЦ ОЛВМ: $y 21=X 1$ (СОТС), $y 38=X 2$ (СПС), $y 61=X 3$ (СКУД), $y 73=X 4$ (СОТ), $y 82=X 5$ (СУЖ), $y 86=X 6$ (СПД), $y 90=X 7$ (ССОИ), характеризующие случайные события готовности/неготовности подсистем ИСБ к выполнению своих целевых функций; 


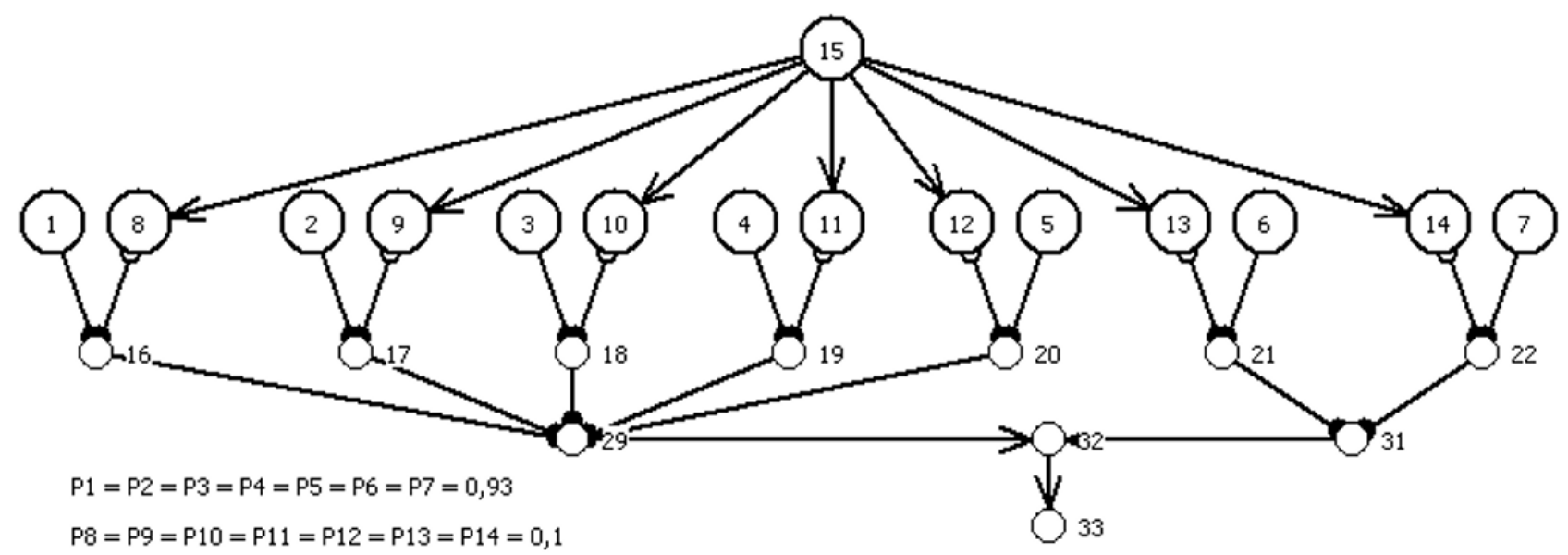

$\mathrm{P} 15=0,7$

Рис. 9. СФЦ структурной устойчивости ИСБ

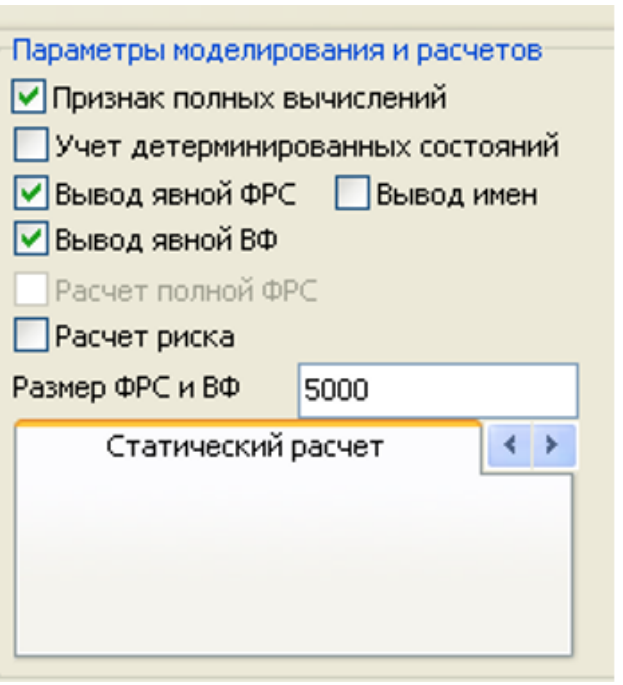

Рис. 10. Параметры моделирования устойчивости ИСБ в ПК «АРБИТР»

2) введем в СФЦ структурной устойчивости ИСБ функциональную вершину $X 15$, которая характеризует случайное событие возникновение/не возникновение поражающего фактора (нарушителя), и обеспечивает вершины $X 8-X 14$, характеризующие случайные события независимого поражения элементов $X 1-X 7$ соответственно.

Полученная СФЦ структурной устойчивости ИСБ приведена на рис. 9

На рис. 9 на выходе фиктивной вершины 16 представлено условие сохранения работоспособности (и не отказа, и не поражения) элемента 1:

$$
y 6=y 1 \cdot \overline{y 8} .
$$

На выходе фиктивных вершин 17-22 представлены аналогичные условия сохране- ния работоспособности элементов 2-7 соответственно. На выходе фиктивной вершины 33 представлено условие сохранения работоспособности хотя бы одним элементом, которое является логическим критерием $Y_{c}=y 33$ комплексной устойчивости рассматриваемой ИСБ в целом:

$$
y 33=y 29 \vee y 31 .
$$

Введем полученную СФЦ структурной устойчивости ИСБ в ПК «АРБИТР» и зададим следующие параметры моделирования, как показано на рис. 10.

В результате моделирования получены:

1) логическая функция устойчивости ИСБ, которая содержит 4 конъюнкции:

$$
Y_{y c m}=\overline{x 13} \cdot \overline{x 14} \cdot x 6 \cdot x 7 \mathrm{~V}
$$

$\vee \overline{x 8} \cdot \overline{x 9} \cdot \overline{x 10} \cdot \overline{x 11} \cdot \overline{x 12} \cdot x 1 \cdot x 2 \cdot x 3 \cdot x 4 \cdot x 5 \vee$

$\vee \overline{x 15} \cdot x 6 \cdot x 7 \vee \overline{x 15} \cdot x 1 \cdot x 2 \cdot x 3 \cdot x 4 \cdot x 5$

2) вероятностная функция устойчивости ИСБ, которая содержит 6 одночленов:

$$
P_{y c m}=Q 15 P 1 P 2 P 3 P 4 P 5+Q 15 P 6 P 7+
$$
+ Q8 Q9 Q10Q11Q12 P15 P1 P2 P3 P4 P5 +

$$
\text { +Q13 Q14 P15 P6 P7 - }
$$

Q8 Q9 Q10 Q11Q12Q13Q14× $\times P 15 P 1 P 2 P 3 P 4 P 5 P 6 P 7-$

$$
\text { - Q15 P1 P2 P3 P4 P5 P6 P7. }
$$

После подстановки исходных данных, а именно: $\quad P 1=P 2=P 3=P 4=P 5=P 6=P 7=$ $=0,93$ (согласно [1] коэффициент готовности, а равно и вероятность реализации целевой 


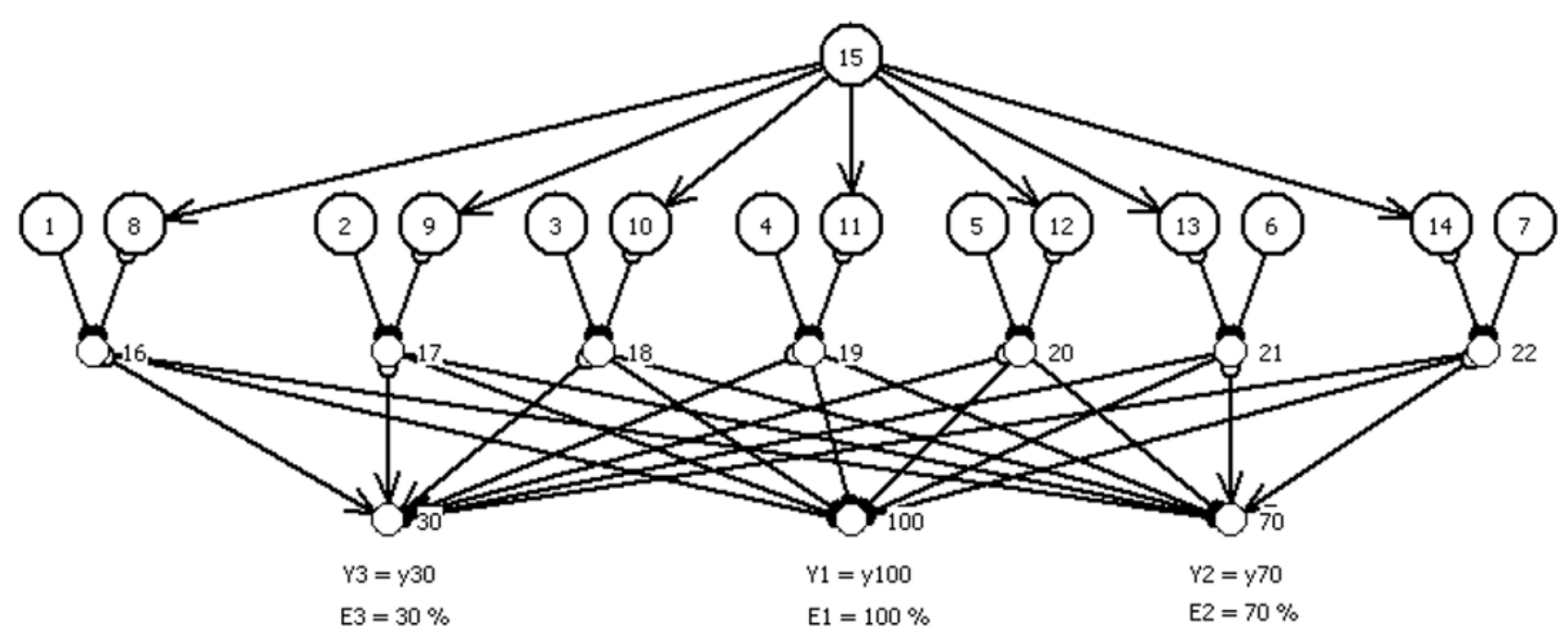

Рис. 11. СФЦ эбфективности ИСБ

функции каждой подсистемой ИСБ, должен быть равен не менее 0,93$) ; P 8=P 9=P 10=$ $=P 11=P 12=P 13=P 14=0,1 \quad$ (для примера); $P 15=0,7$ (для примера), получим вероятность реализации критерия $y 33$, а равно и вероятностный показатель устойчивости ИСБ: $P_{y c m}=0,8642$.

Действительно, разработанная СФЦ устойчивости ИСБ (см. рис. 9), а также ее логическая и вероятностная функции являются немонотонными, что не противоречит положениям ОЛВМ $[12,13]$.

\section{МОДЕЛИРОВАНИЕ И РАСЧЕТ КОМПЛЕКСНОГО ПОКАЗАТЕЛЯ ЭФФЕКТИВНОСТИ ИСБ}

Согласно [15] для объектов вида «вида II», т. е. для объектов, которые могут находиться в некотором числе $j=1,2, \ldots, M$ частично неработоспособных состояний (состояний частичного отказа и/или поражения) $Y_{j}$, определяют комплексный показатель - «коэффициент сохранения эффективности» $\left(K_{э \phi}\right)$, при расчете которого сохраняются общие принципы расчета надежности объектов «вида I», но каждому состоянию объекта, определяемому совокупностью состояний его элементов или каждой возможной его траектории в пространстве состояний элементов, должно быть поставлено в соответствие определенное значение доли сохраняемой номинальной эффективности от 0 до 1 (для объектов «вида I» эффективность в любом состоянии может принимать только два возможных значения: 0 или 1).

В [13] предложено брать в расчет три качественно различных несовместных области состояний, соответствующих эффективностям системы $E_{1}=100 \%, E_{2}=70 \%, E_{3}=30 \%$. Используем в нашем исследовании данный подход.

С учетом ранее разработанной модели устойчивости ИСБ положим, что общее условие ее работоспособности $Y_{c}=y 33=y 29 \vee y 31$ характеризуется тремя качественно различными несовместными областями состояний:

1) состояния полной работоспособности $Y_{1}$, при котором эффективность ИСБ $E_{1}=100 \%$ :

$$
Y_{1}=y 100=y 16 \cdot y 17 \cdot y 18 \cdot y 19 \cdot y 20 \cdot y 21 \cdot y 22
$$

2) состояния частичной работоспособности $Y_{2}$, при котором эффективность ИСБ $E_{2}=70 \%$ :

$Y_{2}=y 70=y 16 \cdot y 17 \cdot y 18 \cdot y 19 \cdot y 20 \cdot(\overline{y 21} \vee \overline{y 22})$

3) состояния частичной работоспособности $Y_{3}$, при котором эффективность ИСБ $E_{3}=30 \%$ :

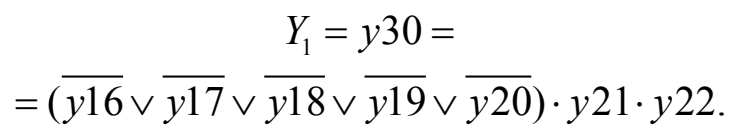

На основании предложенных трех качественно различных несовместных областей состояний получена СФЦ эффективности ИСБ (см. рис. 8). 
Моделирование и оценка эффективности интегрированных систем безопасности объектов ...

Для визуализации трех качественно различных несовместных областей состояний в разрабатываемую СФЦ эффективности введено три фиктивные вершины с номерами 100, 70 и 30 соответственно, на выходах которых представляются критерии функционирования ИСБ с различными уровнями эффективности в $100 \%, 70 \%$ и $30 \%$ соответственно.

Введем полученную СФЦ эффективности ИСБ (с теми же вероятностными показателями элементов) в ПК «АРБИТР». В результате проведения вычислительного эксперимента получим: $\quad p\left(Y_{1}\right)=0,382, \quad p\left(Y_{2}\right)=0,1143$, $p\left(Y_{3}\right)=0,3679$.

Автоматизированное моделирование и расчет показателя эффективности в ОЛВМ осуществляются на основе составного критерия [13]:

$$
K_{э \phi}=W=E_{1} p\left(Y_{1}\right)+E_{2} p\left(Y_{2}\right)+E_{3} p\left(Y_{3}\right) .
$$

Таким образом, определим комплексный показатель эффективности ИСБ:

$$
\begin{aligned}
K_{\text {эф.ИСБ }} & =100 \% \cdot 0,3820+70 \% \cdot 0,1143+ \\
& +30 \% \cdot 0,3679=57,24 \% .
\end{aligned}
$$

Полученные результаты позволяют дополнительно определить вероятность нахождения ИСБ в устойчивом состоянии:

$$
\begin{gathered}
P_{y c m}=p\left(Y_{1}\right)+p\left(Y_{2}\right)+p\left(Y_{3}\right)= \\
=0,3820+0,1143+0,3679=0,8642,
\end{gathered}
$$

которая очевидно совпадает с полученным ранее значением устойчивости ИСБ.

Действительно, разработанная СФЦ эффективности ИСБ (см. рис. 11), а также ее логическая и вероятностная функции являются немонотонными, что не противоречит положениям ОЛВМ [12] и результатам опубликованным ранее в тестовых примерах [13].

\section{ЗАКЛЮЧЕНИЕ}

Современные ИСБ в зависимости от структуры могут быть как системами 1-го типа (все выходные функции характеризуются только двумя уровнями состояний - полной работоспособности, безопасности или полного отказа, аварии), так и системами 2-го типа (качественно-сложными). В качественно-сложных
ИСБ, строящихся в основном для объектов, подлежащих обязательной государственной охране, и других особо важных объектов, имеются выходные функции, характеризующиеся (кроме указанных двух состояний) еще некоторым числом состояний частичного отказа или частичной работоспособности, в которых цели работы системы реализуются с разной степенью эффективности.

В статье предложено использовать общий логико-вероятностный метод для разработки соответствующих моделей и расчета количественных показателей эффективности ИСБ. Произведен вычислительный эксперимент, построены схема функциональной целостности, логическая и вероятностная модели эффективности ИСБ, получен комплексный показатель эффективности с учетом положений ГОСТ 27.301-95.

В результате проведенного исследования, можно сделать вывод о применимости изложенной методики для количественной оценки эффективности ИСБ объектов, подлежащих обязательной государственной охране.

\section{СПИСОК ЛИТЕРАТУРЫ}

1. ГОСТ Р 53704-2009. Системы безопасности комплексные и интегрированные. Общие технические требования.

2. Рогожин А. А. Основы построения интегрированных систем безопасности : учеб. пособие. - Воронеж : Воронежский институт МВД России, 2012. - 74 с.

3. Дурденко В. А., Рогожин А. А. Разработка классификации и архитектуры построения интегрированных систем безопасности // Вестник Воронеж. гос. ун-та. Сер. Системный анализ и информационные технологии. 2013. - № 1. - С. 61-70.

4. Юсупов Р. М., Мусаев А. А. Особенности оценивания эффективности информационных систем и технологий // Труды СПИИРАН. - 2017. - Вып. 51. - С. 5-34.

5. Рогожин А. А., Дурденко В. А., Баторов Б. О. Критерии оценок эффективности систем физической защиты // В сборнике: Информатика: проблемы, методология, технологии: материалы XIV Международной 


\section{В. А. Дурденко, А. А. Рогожин, Б. О. Баторов}

научно-методической конференции. - Воронеж : Воронежский государственный университет, 2014. - Т. 1. - С. 54-58.

6. Панин О. А. Проблемы оценки эффективности функционирования систем физической защиты объектов // Безопасность. Достоверность. Информация. - 2007. № 72. - С. 22-27.

7. Панин О. А. Анализ эффективности интегрированных систем безопасности: принципы, критерии, методы // Системы безопасности. - М. : Гротек, апрель-май 2006. - С. 60-62.

8. Членов А. Н., Климов А. В. Методика оценки эффективности системы безопасности объектов дистанционного банковского обслуживания // Технологии техносферной безопасности. - 2015. -№ 2 (60). - С. 205-211.

9. Панин О. А. Как измерить эффективность? Логико-вероятностное моделирование в задачах оценки систем физической защиты // Безопасность. Достоверность. Информация. - 2008. - № 77. - С. 20-24.

10. Мосолов А. С. Оценка эффективности системы безопасности на основе метода $\mathrm{MoH}$ те-Карло // Системы безопасности. - 2014. № 1. - C. 74-77.

Дурденко Владимир Андреевич - д-р техн. наук, профессор кафедры информационных технологий управления, Воронежский государственный университет.

Тел.: (473) 228-11-60

E-mail: dva_viis@mail.ru

Рогожин Александр Александрович - канд. техн. наук, преподаватель кафедры информационной безопасности, Краснодарский университет МВД России.

Тел.: (961) 523-69-64

E-mail: raa_tsbs@list.ru

Баторов Батор Октябрьевич - адъюнкт кафедры информационных технологий, Академия управления МВД России.

Тел.: (983) 435-31-33

E-mail: 03bator@rambler.ru
11. Рогожин А. А. Применение технологии автоматизированного структурно-логического моделирования для количественной оценки надежности интегрированных систем безопасности: формализованная постановка задачи // Вестник Воронежского института МВД России. - 2013. - № 2. - С. 195-206.

12. Можаев А. С. Теоретические основы общего логико-вероятностного метода автоматизированного моделирования систем / А. С. Можаев, В.Н. Громов. - Санкт-Петербург : ВИТУ, 2000. - 145 с.

13. Пример 1. Устойчивость, технический риск и эффективность двухэлементной дублированной системы [Электронный ресурс]. Режим доступа: https://szma.com/primer1.pdf.

14. Можаев А. С., Можаева И. А. Программный комплекс «АРБИТР» (ПК АСМ СЗМА) // Свидетельство о государственной регистрации программы для ЭВМ от 22.09.2017 № 2017660507.

15. ГОСТ 27.301-95. Надежность в технике. Расчет надежности. Основные положения.

Durdenko Vladimir A. - Doctor of Technical Sciences, Professor, Department of information technology management, Voronezh State University.

Tel.: (473) 228-11-60

E-mail: dva_viis@mail.ru

Rogozhin Alexander A. - Candidate of Technical Sciences, Lecturer, Department of information security, Krasnodar university of the Ministry of the Interior of Russia.

Tel.: (961) 523-69-64

E-mail: raa_tsbs@list.ru.

Batorov Bator O. - postgraduate student, Department of information technology, Academy of management of the Ministry of the Interior of Russia.

Tel.: (983) 435-31-33

E-mail: 03bator@rambler.ru 